\title{
Focused Ion Beam in the Study of Biomaterials and Biological Matter
}

\author{
Kathryn Grandfield and Håkan Engqvist \\ Department of Engineering Sciences, The Angström Laboratory, Uppsala University, Uppsala 751 21, Sweden \\ Correspondence should be addressed to Kathryn Grandfield, kathryn.grandfield@angstrom.uu.se
}

Received 20 April 2011; Accepted 20 June 2011

Academic Editor: David D. Cohen

Copyright ( $) 2012$ K. Grandfield and H. Engqvist. This is an open access article distributed under the Creative Commons Attribution License, which permits unrestricted use, distribution, and reproduction in any medium, provided the original work is properly cited.

\begin{abstract}
The application of focused ion beam (FIB) techniques in the life sciences has progressed by leaps and bounds over the past decade. A once dedicated ion beam instrument, the focused ion beam today is generally coupled with a plethora of complementary tools such as dual-beam scanning electron microscopy (SEM), environmental SEM, energy dispersive X-ray spectroscopy (EDX), or cryogenic possibilities. All of these additions have contributed to the advancement of focused ion beam use in the study of biomaterials and biological matter. Biomaterials, cells, and their interfaces can be routinely imaged, analyzed, or prepared for techniques such as transmission electron microscopy (TEM) with this comprehensive tool. Herein, we review the uses, advances, and challenges associated with the application of FIB techniques to the life sciences, with particular emphasis on TEM preparation of biomaterials, biological matter, and their interfaces using FIB.
\end{abstract}

\section{Challenges with Biological Samples}

Analyzing cellular and biological structures at high resolution remains a challenge due to the nature of biological materials and their interaction with electron and ion beams. Nonconductive samples simply result in charging, which limits the ability to image them with ease or high quality. Besides the use of an environmental chamber, solutions exist to mediate these problems.

Primarily, a sequence of sample preparation steps aids in the reduction of artefacts associated with biological matter. The fixation, dehydration, and embedding of samples are a standard protocol for many researchers. Indeed, these methods present potential drawbacks. Firstly, it is unclear how much of the original structure is altered after preservation techniques. The penetration of chemical fixatives such as aldehydes and osmium tetroxide indeed influences the chemical make-up of the sample as a whole. On the other hand, the addition of heavy elements such as osmium through OTOTO (osmium tetroxde/thiocarbohydrazide/osmium tetroxide/thiocarbohydrazide/osmium tetroxide) conductive staining has been shown to lead to a reduction in charging artefacts, simplifying the imaging process $[1,2]$. Nevertheless, subsequent embedding steps are associated with slight sample heating and shrinkage, which may alter natural structures. In addition, the cutting and grinding of embedded specimens may introduce additional damage to surfaces analyzed. In the case of biomaterials in contact with tissues there exists a so-called hybrid interface of soft and hard materials in contact. Embedding and sectioning techniques may result in the elimination of interfacial contact. Palmquist et al. have furthermore demonstrated that the choice of embedding resin has an effect on the overall shrinkage and separation of the specimen [3]. Nevertheless, in some cases, embedding of biological samples has been proven to yield higher quality results, such as improved imaging of subcellular components versus critical-point dried samples, which tend to yield better information regarding external cellular structures [2].

To circumvent the artefacts that may be associated with conventional fixative preparation methods, a cyrogenic (cryo-) approach may be taken. Modern focused ion beam systems are implementing cryostages and transfer devices to eliminate the preservation steps usually required for biological specimens. In this regard, the true, unaltered 
structure of cells and tissues may be analyzed and prepared for further investigations. Lamers et al. have shown that compared to conventional FIBSEM techniques, cryogenic FIBSEM of cells on nanopatterned interfaces preserves the extracellular matrix and details such as cell membranes, offering an improved cellular contrast [4]. Challenges indeed still remain, such as the deposition of protective platinum and elimination of frost buildup on sample surfaces as experienced by Edwards et al. during the preparation of mesenchymal stem cells on titanium [5]. Hayles et al. have demonstrated a method for remedying the difficulties of platinum deposition at low temperature, whereby reducing the source heating temperature and tilting the sample towards the Gas Injector System (GIS) enables better control of coating deposition and uniformity [6]. As cryo-FIBSEM preparation of biological samples for TEM becomes more widespread, with it we will see the emergence of additional techniques to improve sample quality and yield.

\section{Functionalized Analysis Using FIB}

The most dramatic improvement to the focused ion beam system has been the addition of a secondary column, an electron column, to create the dual beam FIBSEM instrument. This system, which has both ion and electron beam focused in coincidence on the sample, has enabled simultaneous milling and imaging of samples.

Cross-sectional analysis is of great interest to visualize the interaction between tissues/cells and substrates. To improve image quality, de Winter et al. have demonstrated the success of milling a U-shaped trench around the cross section of interest, shown in Figure 1. Removing surrounding material has limited shadowing effects and improved uniformity in the cross-section contrast [1]. As in TEM sample preparation, the deposition of a protective surface layer is recommended. Materials commonly deposited include platinum and carbon and have been shown to reduce the socalled curtaining effect [9]. Other imaging techniques such as the use of back-scattered electrons have provided more in-depth information on the chemical constructs of cellular systems [10]. Furthermore, the investigation of cell adhesion to surfaces is of great interest. Use of the FIB to create and analyze cells in contact with nano-patterned surfaces has demonstrated the affinity of cells and proteins for high-density nano- and micropatterned surfaces [7, 11, 12]. Friedmann et al. have demonstrated FIBSEM for the analysis of cell in-growth into porous alumina membranes and shown the penetration of microneedles through cell mem-branes using a pie-slice cutting method, illustrated in Figure 2 [7].

In addition to electron imaging, a number of other technologies have been incorporated into the FIB to enhance analytical power. Elemental analysis with EDX on cross-sectional samples can provide additional information. In the case of Lešer et al., this analysis provided the general composition of digestive gland cells, regardless of the complications associated with X-ray production and the tilt of the sample surface towards the detector [10].
An important advancement in improving the efficiency of FIB work has been the development of automation. With programs such as FEI's Slice and View software, and similar programs offered by other manufacturers, automated and sequential milling of samples reduces user and instrument time while enabling a three-dimensional investigation of the sample. Three-dimensional structural analysis has been demonstrated on biomaterial tissue interfaces, such as in the study by Giannuzzi et al. [8]. Tomograms of the TiUnite dental implant surface and bone, shown in Figure 3, confirmed and mapped the bone-implant contact in three dimensions [8]. On a cellular level, FIB tomography is a complement to frequently employed electron tomography of cells, offering information on a lower resolution, yet of a larger field of view $[13,14]$.

\section{FIB for Biological TEM Preparation}

The conventional method for preparation of TEM samples in the life sciences has always been ultramicrotomy. Recently, cryogenic ultramicrotomy has also been introduced to limit the need for sample fixation. However, both forms of ultramicrotomy present significant drawbacks, mainly in their inability to maintain true structural features. It is well known that cellular structures are generally compressed in the direction of cutting. Marko et al. have clearly shown that, using FIB preparation methods, this distortion can be eradicated [15]. The second main hindrance to ultramicrotomy preparation lies in the inability to choose specific sites of interest. However, with a dual-beam FIBSEM system, specific sites can be targeted.

Focused ion beam is routinely used for the production of TEM samples for higher resolution analysis. The unique system enables the production of TEM lamellae from bulk samples. A variety of forms of samples can be produced including cross-section, plan view, H-bar, ex situ, or direct liftout, covered in more detail by Phaneuf et al., Giannuzzi et al. and $\mathrm{Li}$ et al. [16-18]. Of course, depending on the sample, a number of specialized methods for preparation may be employed, such as the case in Wright et al.'s where a hydroxyapatite particle on a TEM grid was milled to a create a thin electron transparent ledge or alternatively embedded in silver dag and applied to a grid and then thinned [19]. Embedding the particles in silver dag in fact reduced the charging of the nonconductive particles, and similar embedding techniques could be of interest for this purpose [19].

Preparation of TEM samples with FIB has been simplified with the advent of the in situ lift-out method. In the preparation of ivory dentine for TEM, use of the in-situ method resulted in a much higher sample yield than the ex situ liftout method, in which electrostatic forces between the glass needle and sample caused the repulsion of many thinned lamellae [20].

Generally, a higher-quality sample is prepared with FIB. However, in the case of tooth, focused ion beam samples appeared to show distorted or fuzzy features in TEM resulting from the amorphization of the prepared sample [21]. 


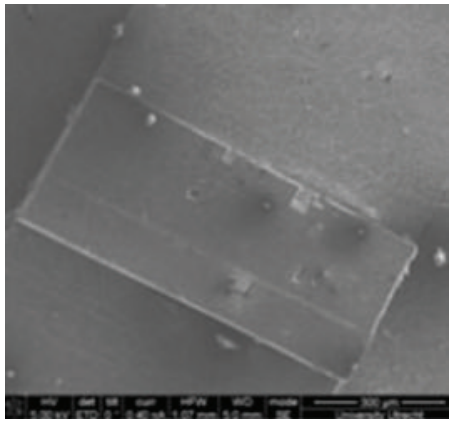

(a)

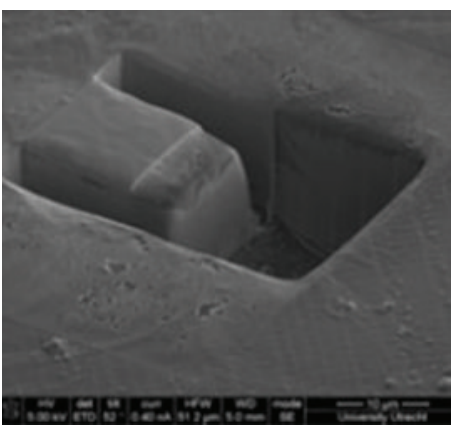

(d)

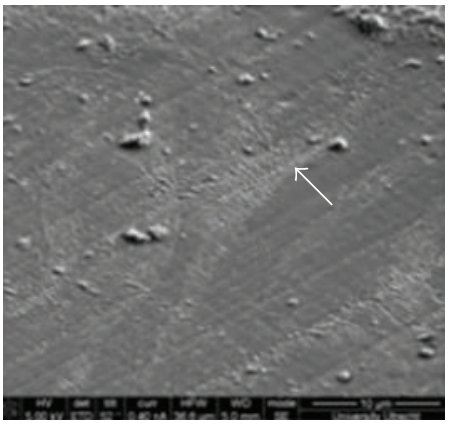

(b)

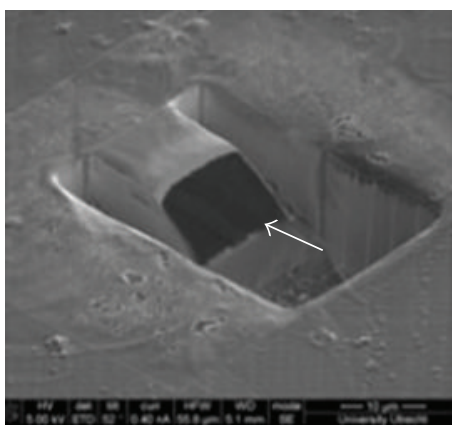

(e)

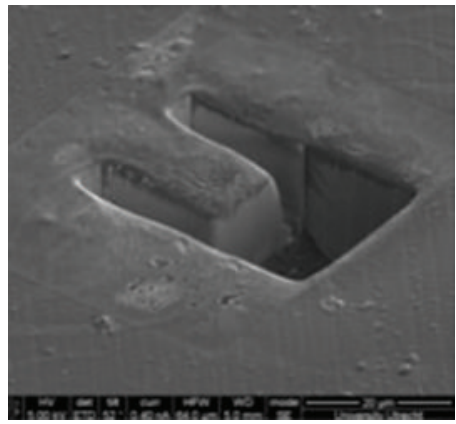

(c)

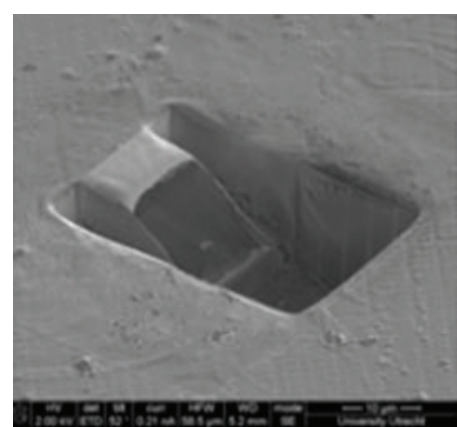

(f)

FIgURE 1: The slice and view geometry used by De Winter et al. The U-Shaped trench around the surface of interest improved contrast uniformity and reduced shadowing effects, reproduced with permission from [1].

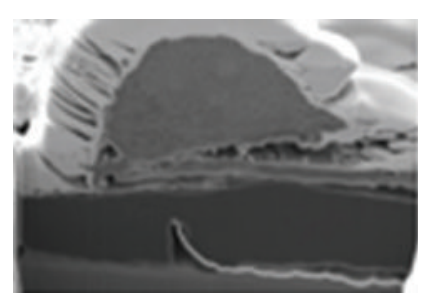

(a)

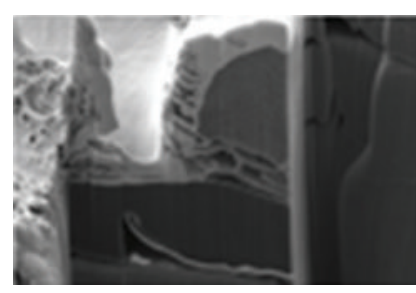

(b)

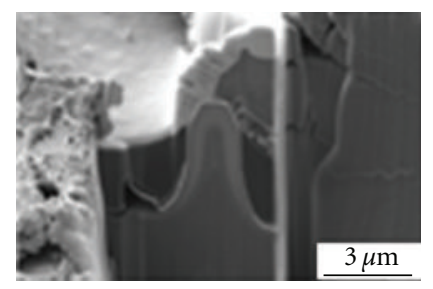

(c)

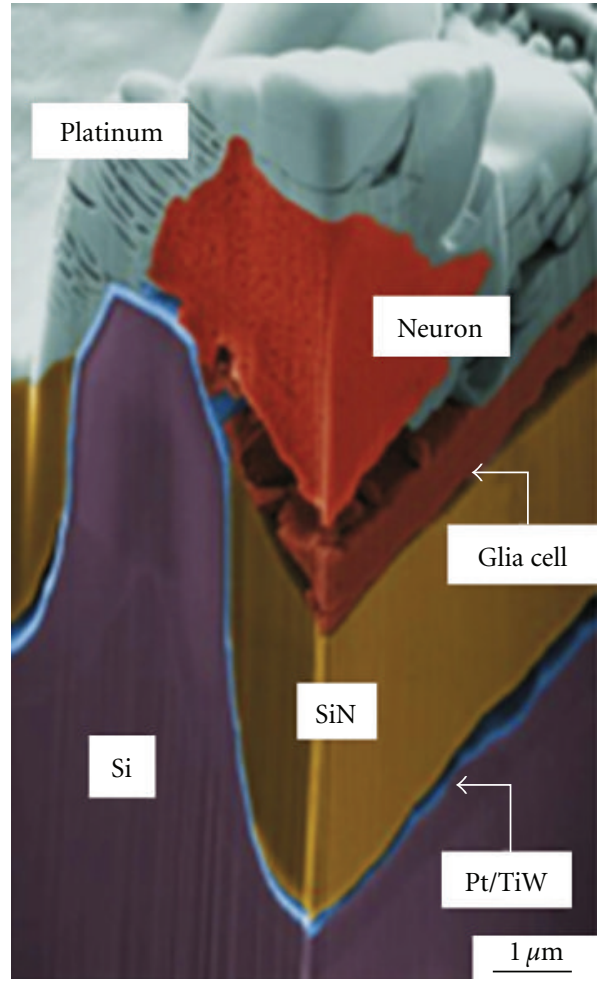

(d)

Figure 2: The unique pie-slice cutting method enables visualization of cocultivated primary murine glia cells and neurons on a microneedle array, reproduced with permission from [7]. 


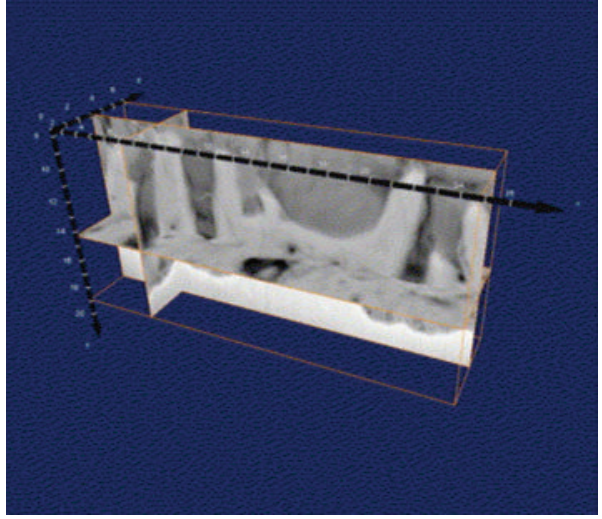

(a)

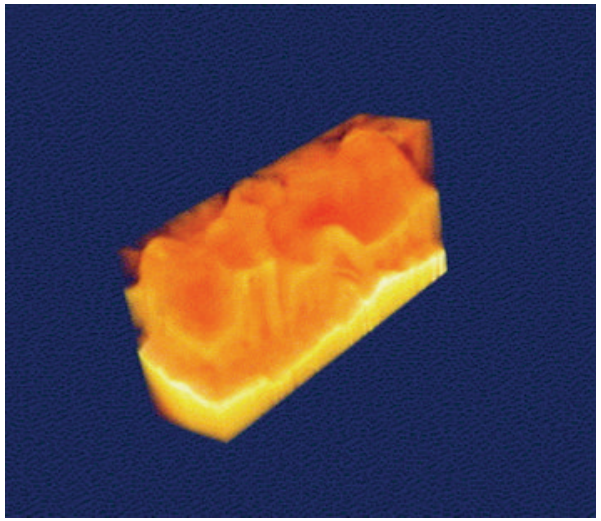

(c)

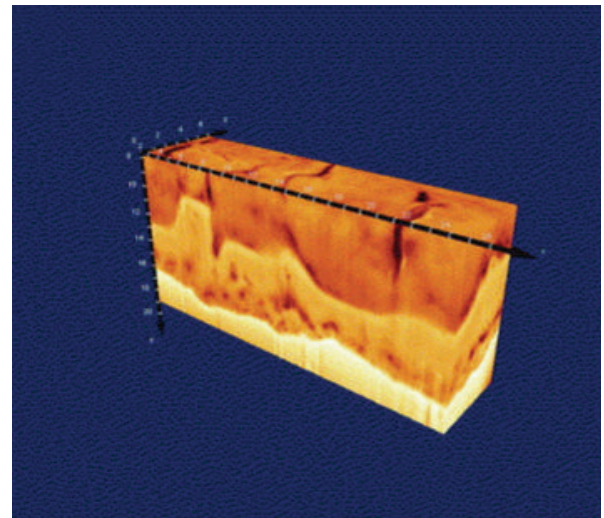

(b)

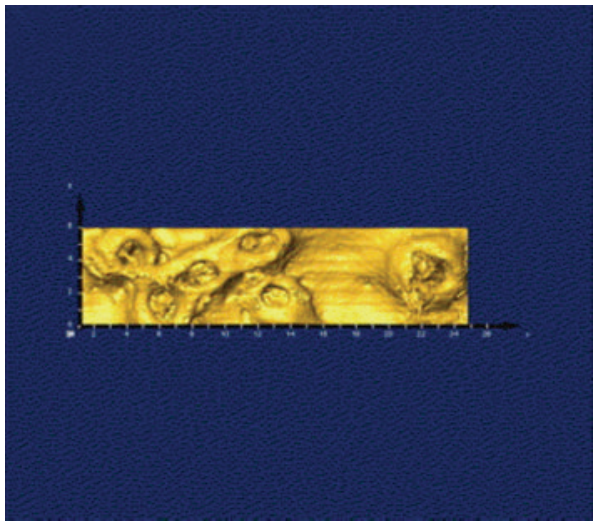

(d)

FIgURE 3: Three-dimensional reconstructions of the TiUnite and bone interface using Slice and View FIB, reproduced with permission from $[8]$.

In that particular case, ultramicrotomed samples showed an improved morphology. It is important to note, however, that sample amorphization is generally limited to the first $10 \mathrm{~nm}$ of the sample surface and can be reduced by implementing low-energy ion bombardment during the final milling stages or as a "cleaning" stage [22]. As an example, reducing FIB milling voltages to $2 \mathrm{keV}$ in final milling stages reduced the amorphized regions of Si to $2 \mathrm{~nm}$ from the significantly larger $22 \mathrm{~nm}$ damaged region seen with milling at $30 \mathrm{keV}$, thereby greatly improving the quality of sample produced $[23,24]$.

Techniques and protocols exist to increase the TEM sample yield, as this is always the aim in TEM sample preparation. While many claim that sample preparation times may be reduced to less than an hour, sample preparation in reality depends on the experience of the operator and the maintenance of the instrument. Each operator, and each sample type have their own tips and tricks for success. In general, however, it is always recommended to protect the sample surface with electron-beam-deposited platinum before irradiating the surface of interest with the ion beam. A layer of protective metal has also been shown to prevent unnecessary ion implantation in cells and improve milling quality perpendicular to the beam [25]. In our experiences, a lowering of the sample stage followed by placement of the micromanipulator probe at eucentric height, then subsequent replacement of the sample stage has resulted in less instances of unwanted probe-sample contact and destruction of lamellae prior to liftout. In many of our samples, a hard material such as zirconia or stainless steel is interfaced to the much softer bone tissue. In these instances, there will be a differential in milling rates, sometimes requiring extra milling over solely the hard material [26]. An example of this is shown in Figure 4, during the preparation of a hydroxyapatite ceramic scaffold interfacing human bone. Pillars of the hydroxyapatite material remain, while the bone is milled away much faster. With continued milling, the final result shows a uniformly thin specimen. For these interfacial specimens, FIB avoids introducing stresses over the contact zone, or breakage due to variance in mechanical strength, as what often occurs with ultramicrotomy of hard implants interfacing soft tissue or cells. Due to its ability to maintain interfacial integrity, the use of focused ion beam for TEM sample preparation is becoming the dominant method in biomaterials investigations and has been success-fully applied to a number of biomaterials and interfaces including titanium and HA-coated titanium implants [27-29], titanium to bone $[30,31]$, hydroxyapatite to bone $[26,32]$, hydroxyapatite to cells [33], calcium aluminate to bone [34], 


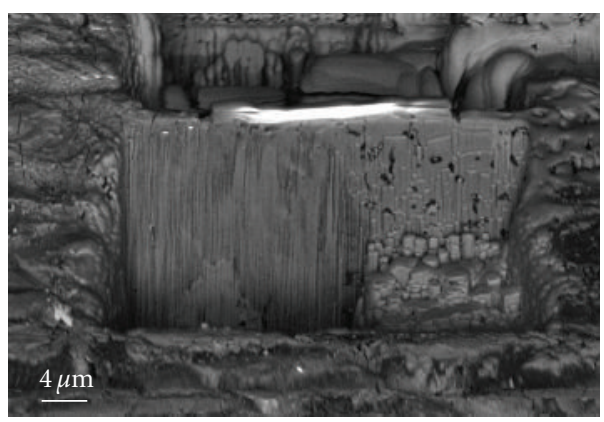

(a)

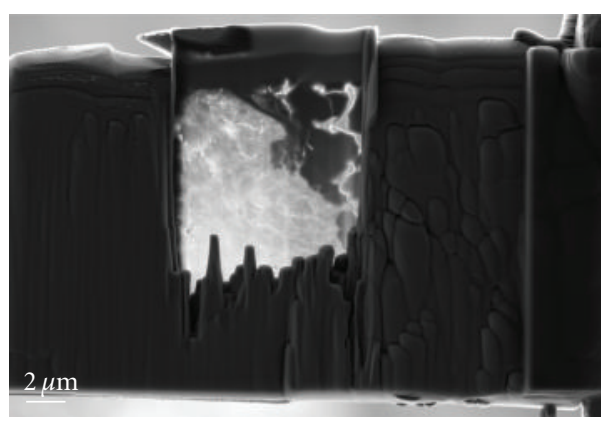

(b)

FIGURE 4: TEM sample preparation of a hydroxyapatite scaffold (right portion of sample) interfacing to human bone (left portion of sample) by FIB. Pillars of remaining material due to differential milling rates are seen in (a), while the final sample can be thinned to uniform thickness, (b), with continued milling.

cobalt chromium to bone [35], and glass ionomer cements to dentin [21].

\section{Conclusions and Future Outlook}

The focused ion beam instrument has revolutionized the way in which biological samples are investigated. Highresolution structural and chemical information is attainable from FIB-prepared TEM samples that retain their interfacial contact, or are undeformed in their cellular structure. Interactions between cells and surfaces, which are vital for the understanding and subsequent development of biomaterials, are visible now from the cell interior. Three-dimensional understanding of structures and interfaces is made possible with the dual beam FIBSEM systems. Finally, advances such as the addition of cryocapabilities are enabling the analysis of biological matter in as much of an unchanged state as possible, adding new information to previously investigated specimens.

In the future, the ability to process and analyze biological samples from start to finish, at any length scale, in the focused ion beam is a reality. Properly maintained and sufficiently equipped instruments may enable this. In the history of FIB development, we have noted the shift in usage from primarily microelectronics and materials science based, to a user community that encompasses many areas including the life sciences. The challenge in the next phase of FIB development then shifts to, in what other ways can we use the FIB? What other devices can we couple with the instrument or what changes can be made to provide answers to our scientific questions? In the next decade, we will surely see the continued emergence of new focused ion beam applications and advances.

\section{Acknowledgments}

Financial support is gratefully acknowledged from the VINNOVA VinnVäxt Program of Sweden and the Institute for Biomaterials and Cell Therapy, Gothenburg, Sweden.

\section{References}

[1] D. A. M. De Winter, C. T. W. M. Schneijdenberg, M. N. Lebbink et al., "Tomography of insulating biological and geological materials using focused ion beam (FIB) sectioning and low-kV BSE imaging," Journal of Microscopy, vol. 233, no. 3, pp. 372-383, 2009.

[2] V. Lešer, D. Drobne, Z. Pipan, M. Milani, and F. Tatti, "Comparison of different preparation methods of biological samples for FIB milling and SEM investigation," Journal of Microscopy, vol. 233, no. 2, pp. 309-319, 2009.

[3] A. Palmquist, F. Lindberg, L. Emanuelsson, R. Brånemark, H. Engqvist, and P. Thomsen, "Morphological studies on machined implants of commercially pure titanium and titanium alloy (Ti6Al4V) in the rabbit," Journal of Biomedical Materials Research. Part B, vol. 91, no. 1, pp. 309-319, 2009.

[4] E. Lamers, X. F. Walboomers, M. Domanski et al., "Cryo dualbeam focused ion beam-scanning electron microscopy to evaluate the interface between cells and nanopatterned scaffolds," Tissue Engineering. Part C, vol. 17, no. 1, pp. 1-7, 2010.

[5] H. K. Edwards, M. W. Fay, S. I. Anderson, C. A. Scotchford, D. M. Grant, and P. D. Brown, "An appraisal of ultramicrotomy, FIBSEM and cryogenic FIBSEM techniques for the sectioning of biological cells on titanium substrates for TEM investigation," Journal of Microscopy, vol. 234, no. 1, pp. 16-25, 2009.

[6] M. F. Hayles, D. J. Stokes, D. Phifer, and K. C. Findlay, "A technique for improved focused ion beam milling of cryoprepared life science specimens," Journal of Microscopy, vol. 226, no. 3, pp. 263-269, 2007.

[7] A. Friedmann, A. Hoess, A. Cismak, and A. Heilmann, "Investigation of cell-substrate interactions by focused ion beam preparation and scanning electron microscopy," Acta Biomaterialia, vol. 7, no. 6, pp. 2499-2507, 2011.

[8] L. A. Giannuzzi, D. Phifer, N. J. Giannuzzi, and M. J. Capuano, "Two-dimensional and 3-dimensional analysis of bone/dental implant interfaces with the use of focused ion beam and electron microscopy," Journal of Oral and Maxillofacial Surgery, vol. 65, no. 4, pp. 737-747, 2007.

[9] L. A. Giannuzzi and F. A. Stevie, Introduction to Focused Ion Beams, Springer Science, Boston, Mass, USA, 2005.

[10] V. Lešer, M. Milani, F. Tatti, Ž. P. Tkalec, J. Štrus, and D. Drobne, "Focused ion beam (FIB)/scanning electron microscopy (SEM) in tissue structural research," Protoplasma, vol. 246, no. 1, pp. 41-48, 2010. 
[11] V. Raffa, O. Vittorio, V. Pensabene, A. Menciassi, and P. Dario, "FIB-nanostructured surfaces and investigation of bio/nonbio interactions at the nanoscale," IEEE Transactions on Nanobioscience, vol. 7, no. 1, pp. 1-10, 2008.

[12] E. Martínez, E. Engel, C. López-Iglesias, C. A. Mills, J. A. Planell, and J. Samitier, "Focused ion beam/scanning electron microscopy characterization of cell behavior on polymer micro-/nanopatterned substrates: a study of cell-substrate interactions," Micron, vol. 39, no. 2, pp. 111-116, 2008.

[13] F. Schmidt, M. Kühbacher, U. Gross, A. Kyriakopoulos, H. Schubert, and R. Zehbe, "From 2D slices to 3D volumes: image based reconstruction and morphological characterization of hippocampal cells on charged and uncharged surfaces using FIB/SEM serial sectioning," Ultramicroscopy, vol. 111, no. 4, pp. 259-266, 2011.

[14] J. A. W. Heymann, M. Hayles, I. Gestmann, L. A. Giannuzzi, B. Lich, and S. Subramaniam, "Site-specific 3D imaging of cells and tissues with a dual beam microscope," Journal of Structural Biology, vol. 155, no. 1, pp. 63-73, 2006.

[15] M. Marko, C. Hsieh, R. Schalek, J. Frank, and C. Mannella, "Focused-ion-beam thinning of frozen-hydrated biological specimens for cryo-electron microscopy," Nature Methods, vol. 4, no. 3, pp. 215-217, 2007.

[16] M. W. Phaneuf, "Applications of focused ion beam microscopy to materials science specimens," Micron, vol. 30, no. 3, pp. 277-288, 1999.

[17] L. A. Giannuzzi and F. A. Stevie, "A review of focused ion beam milling techniques for TEM specimen preparation," Micron, vol. 30, no. 3, pp. 197-204, 1999.

[18] J. Li, T. Malis, and S. Dionne, "Recent advances in FIB-TEM specimen preparation techniques," Materials Characterization, vol. 57, no. 1, pp. 64-70, 2006.

[19] D. M. Wright, J. J. Rickard, N. H. Kyle et al., "The use of dual beam ESEM FIB to reveal the internal ultrastructure of hydroxyapatite nanoparticle-sugar-glass composites," Journal of Materials Science: Materials in Medicine, vol. 20, no. 1, pp. 203-214, 2009.

[20] V. Jantou, M. Turmaine, G. D. West, M. A. Horton, and D. W. McComb, "Focused ion beam milling and ultramicrotomy of mineralised ivory dentine for analytical transmission electron microscopy," Micron, vol. 40, no. 4, pp. 495-501, 2009.

[21] E. Coutinho, T. Jarmar, F. Svahn et al., "Ultrastructural characterization of tooth-biomaterial interfaces prepared with broad and focused ion beams," Dental Materials, vol. 25, no. 11, pp. 1325-1337, 2009.

[22] N. I. Kato, "Reducing focused ion beam damage to transmission electron microscopy samples," Journal of Electron Microscopy, vol. 53, no. 5, pp. 451-458, 2004.

[23] LA Giannuzzi, R Geurts, and J. Ringnalda, "2 keV Ga+ FIB milling for reducing amorphous damage in silicon," Microscopy and Microanalysis, vol. 11, no. S02, pp. 828-829, 2005.

[24] L. A. Giannuzzi, "Reducing FIB damage using low energy ions," Microscopy and Microanalysis, vol. 12, no. 2, pp. 12601261, 2006.

[25] D. Drobne, M. Milani, V. Lešer, and F. Tatti, "Surface damage induced by FIB milling and imaging of biological samples is controllable," Microscopy Research and Technique, vol. 70, no. 10, pp. 895-903, 2007.

[26] K Grandfield, A Palmquist, F Ericson et al., "Bone response to free-form fabricated hydroxyapatite and zirconia scaffolds: a transmission electron microscopy study in the human maxilla," Clinical Implant Dentistry and Related Research, Feb 11, 2010. In press.
[27] T. Jarmar, A. Palmquist, R. Brånemark, L. Hermansson, H. Engqvist, and P. Thomsen, "Technique for preparation and characterization in cross-section of oral titanium implant surfaces using focused ion beam and transmission electron microscopy," Journal of Biomedical Materials Research. Part A, vol. 87, no. 4, pp. 1003-1009, 2008.

[28] M. Iliescu, V. Nelea, J. Werckmann, and I. N. Mihailescu, "Transmission electron microscopy investigation of pulsedlaser deposited hydroxylapatite thin films prepared by tripod and focused ion beam techniques," Surface and Coatings Technology, vol. 187, no. 1, pp. 131-140, 2004.

[29] F. Lindberg, J. Heinrichs, F. Ericson, P. Thomsen, and H. Engqvist, "Hydroxylapatite growth on single-crystal rutile substrates," Biomaterials, vol. 29, no. 23, pp. 3317-3323, 2008.

[30] A. Palmquist, F. Lindberg, L. Emanuelsson, R. Brånemark, H. Engqvist, and P. Thomsen, "Biomechanical, histological, and ultrastructural analyses of laser micro- and nano-structured titanium alloy implants: a study in rabbit," Journal of Biomedical Materials Research. Part A, vol. 92, no. 4, pp. 1476-1486, 2010.

[31] H. Engqvist, G. A. Botton, M. Couillard et al., "A novel tool for high-resolution transmission electron microscopy of intact interfaces between bone and metallic implants," Journal of Biomedical Materials Research. Part A, vol. 78, no. 1, pp. 2024, 2006.

[32] K. Grandfield, E. A. McNally, A. Palmquist, G. A. Botton, P. Thomsen, and H. Engqvist, "Visualizing biointerfaces in three dimensions: electron tomography of the bone-hydroxyapatite interface," Journal of the Royal Society Interface, vol. 7, no. 51, pp. 1497-1501, 2010.

[33] H. Engqvist, F. Svahn, T. Jarmar et al., "A novel method for producing electron transparent films of interfaces between cells and biomaterials," Journal of Materials Science: Materials in Medicine, vol. 19, no. 1, pp. 467-470, 2008.

[34] A. Palmquist, T. Jarmar, L. Hermansson et al., "Calcium aluminate coated and uncoated free form fabricated cocr implants: a comparative study in rabbit," Journal of Biomedical Materials Research. Part B, vol. 91, no. 1, pp. 122-127, 2009.

[35] K. Grandfield, A. Palmquist, S. Gonçalves et al., "Free form fabricated features on $\mathrm{CoCr}$ implants with and without hydroxyapatite coating in vivo: a comparative study of bone contact and bone growth induction," Journal of Materials Science: Materials in Medicine, vol. 22, no. 4, pp. 899-906, 2011. 

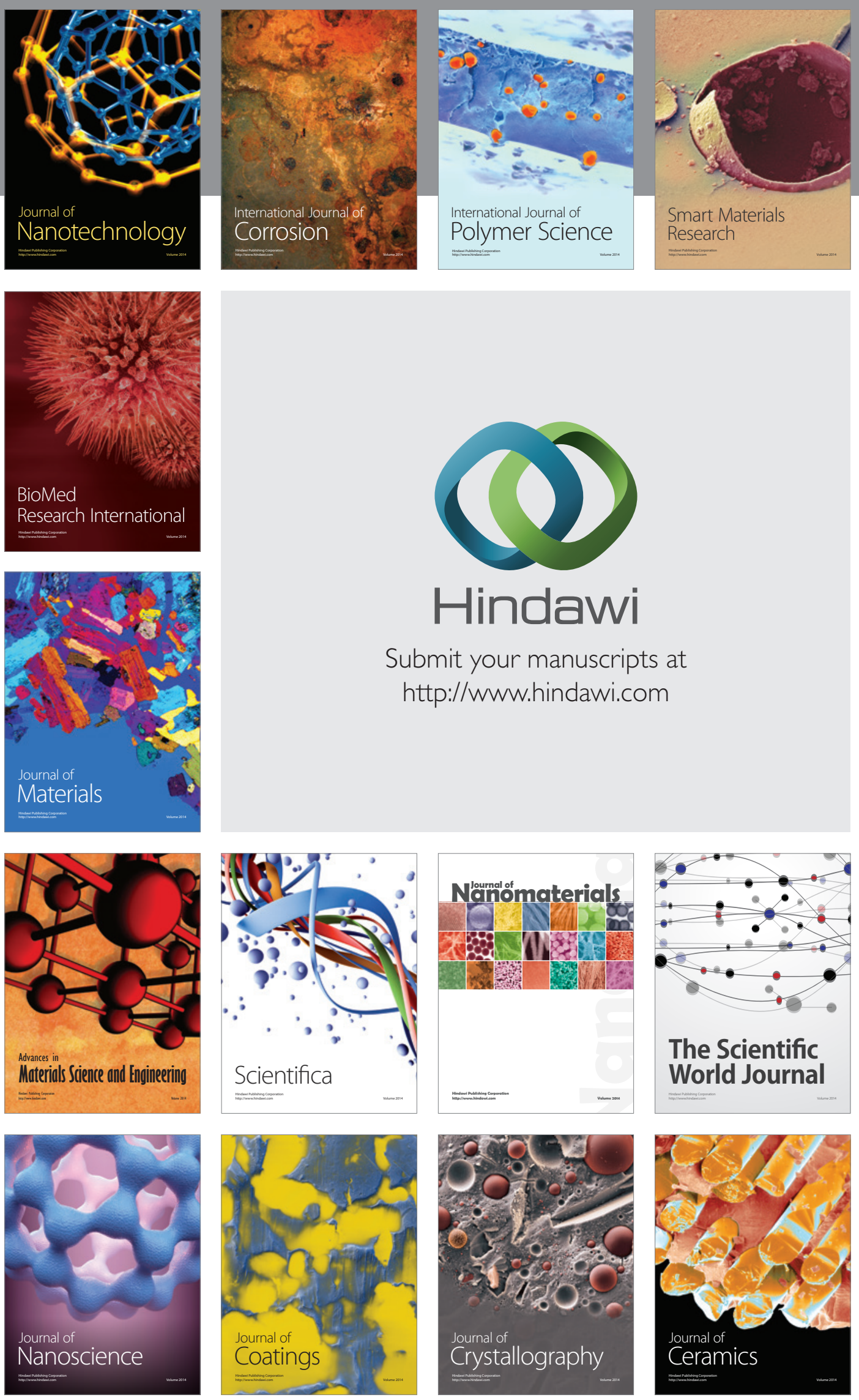

The Scientific World Journal

Submit your manuscripts at

http://www.hindawi.com

\section{World Journal}

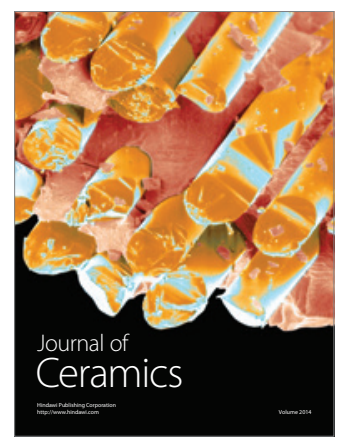

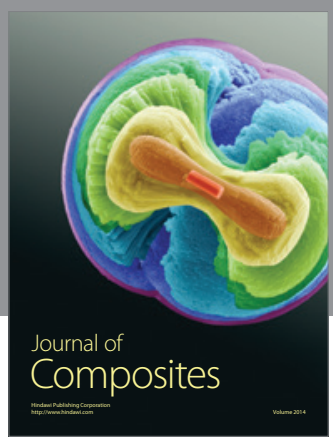
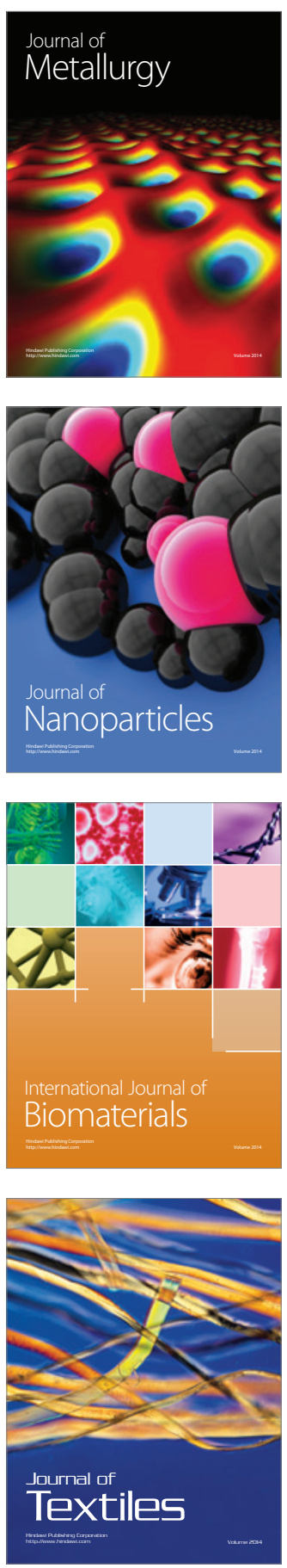